\title{
Phylogenetic relationships of chrysanthemums in Korea based on novel SSR markers
}

\author{
A.A. Khaing ${ }^{1}$, K.T. Moe ${ }^{1}$, W.J. Hong ${ }^{1}$, C.S. Park ${ }^{1}$, K.H. Yeon ${ }^{1}$, H.S. Park ${ }^{2}$, \\ D.C. Kim ${ }^{2}$, B.J. Choi ${ }^{2}$, J.Y. Jung ${ }^{3}$, S.C. Chae ${ }^{4}$, K.M. Lee ${ }^{5}$ and Y.J. Park ${ }^{1,6}$ \\ ${ }^{1}$ Department of Plant Resources, College of Industrial Sciences, \\ Kongju National University, Yesan, Republic of Korea \\ ${ }^{2}$ Chrysanthemum Research Station, Yesan, Republic of Korea \\ ${ }^{3}$ Department of Companion \& Laboratory Animal Science, \\ Kongju National University, Yesan, Republic of Korea \\ ${ }^{4}$ Department of Horticultural Science, Kongju National University, Yesan, \\ Republic of Korea \\ ${ }^{5}$ Department of Plant Science and Techonology, Sangmyung University, \\ Cheonan, Republic of Korea \\ ${ }^{6}$ Legume Bio-Resource Center of Green Manure, \\ Kongju National University, Yesan, Republic of Korea \\ Corresponding author: Y.J. Park \\ E-mail: yjpark@kongju.ac.kr
}

Genet. Mol. Res. 12 (4): 5335-5347 (2013)

Received November 27, 2012

Accepted April 2, 2013

Published November 7, 2013

DOI http://dx.doi.org/10.4238/2013.November.7.8

\begin{abstract}
Chrysanthemums are well known for their esthetic and medicinal values. Characterization of chrysanthemums is vital for their conservation and management as well as for understanding their genetic relationships. We found 12 simple sequence repeat markers (SSRs) of 100 designed primers to be polymorphic. These novel SSR markers were used to evaluate 95 accessions of chrysanthemums (3 indigenous and 92 cultivated accessions). Two hundred alleles were identified, with an average of 16.7 alleles per locus. KNUCRY-77
\end{abstract}


gave the highest polymorphic information content value (0.879), while KNUCRY-10 gave the lowest (0.218). Similar patterns of grouping were observed with a distance-based dendrogram developed using PowerMarker and model-based clustering with Structure. Three clusters with some admixtures were identified by model-based clustering. These newly developed SSR markers will be useful for further studies of chrysanthemums, such as taxonomy and markerassisted selection breeding.

Key words: Chrysanthemum; SSR; Marker development; Genotyping; Population structure

\section{INTRODUCTION}

Chrysanthemums, often called mums or chrysanths, belong to the genus Chrysanthemum and the family Asteraceae (Compositae). Mums are one of the most popular ornamentals in the world. They have diversified growth habits, inflorescence forms, and colors. Demand for chrysanthemum production, including cut flowers, gardens, potted plants, and ground-cover types, has increased worldwide.

In addition to ornamental effects, chrysanthemum flowers have antibacterial, antifungal, and antiviral activities (Yeung, 1983). They also have a calming, antihypertensive effect. Thus, chrysanthemum flowers have been used in traditional Chinese medicine for centuries. White chrysanthemum is considered slightly superior to other forms for nourishing the liver. Yellow chrysanthemum is used most often to treat eye redness and headache (Yeung, 1983). In addition to its medicinal properties, chrysanthemum extract can also be used as raw material in dye and tea production. In particular, simple chrysanthemum flower tea is a very common beverage in China and Korea. Chrysanthemum leaves are steamed or boiled and used as greens in Chinese cuisine. Although chrysanthemums have endless uses, relatively few systematic genetic analyses have been performed compared with other crops.

The genome of mums is composed of multiple sets of chromosomes that range from diploid to decaploid (Hartl and Jones, 2009). Although some pseudo selfincompatible plants have been discovered (Anderson et al., 1992), selfing is generally not possible because of a strong self-incompatibility system, common to members of the family Asteraceae (Richards, 1986). Moreover, a long period of cultivation, natural selection, and artificial crossing has led to numerous cultivars, abundant morphological variation, powerful suitability, wide distribution, and an extremely complex genetic background. Thus, developing a high-resolution technique is important for assessing and distinguishing the more complicated mum accessions.

High levels of polymorphisms at the DNA level in chrysanthemums have been determined (Wolff and Peters-Van Rijn, 1993), and Wolff et al. (1995) found identical DNA patterns in different accessions of the same chrysanthemum cultivar using random amplified polymorphic DNA (RAPD). Huang et al. (2000) detected molecular markers in three hybrid combinations of chrysanthemums using RAPD, and they were able to select 22 primers of the 45 random primers screened. 
Most genetic studies in chrysanthemums have emphasized the ornamental traits of inflorescence, such as color (Li et al., 2005) and floret form (Chen et al., 2003), and vegetative traits, such as plant type (Jiang et al., 2003; Zhao et al., 2009; Zhang et al., 2010a). Flowering is photoperiod-, temperature-, and vernalization-dependent, and its genetic control is expected to be complex. Zhang et al. (2010b) investigated the inheritance of two flowering traits of chrysanthemums, the initial blooming time, and the duration of flowering using segregation within an $\mathrm{F}_{1}$ population derived from a cross between the autumn-flowering "Yuhualuoying" and the summer-flowering "Aoyunhanxiao" cultivars. Their efforts regarding a marker - trait association analysis based on sequence-related amplified polymorphism (SRAP) genotyping uncovered 10 markers for initial blooming time and 12 for duration of flowering. Although a considerable number of DNA markers have been identified, substantially more DNA markers are needed to initiate genetic studies for these plant species.

Simple sequence repeat markers (SSRs) have become one of the most widely used molecular markers for genetic studies, because they offer several advantages, such as technical simplicity, relatively low cost, high genetic resolution power, and high polymorphism. SSRs, or microsatellite markers, have been used to study genetic diversity, phylogenetic relationships, classification, evolutionary processes, and quantitative trait loci in many crops (Dixit et al., 2010; Cho et al., 2010, 2011; Lu and Park, 2012; Moe et al., 2010, 2012; Yoon et al., 2012; Zhao et al., 2012; Khaing et al., 2013). Moreover, they are reliable and easy to score (Gupta and Varshney, 2000). Thus, Moe et al. (2011) constructed an SSR-enriched library using a modified biotin-streptavidin capture method and determined the distribution of SSR motifs in the sequence data of the chrysanthemum library. However, they did not screen with polymerase chain reaction (PCR) amplification for polymorphism with the primers designed. There is a need to screen and confirm the amplification and level of polymorphism to assess whether the designed primers are useful DNA markers for identifying individuals in a population.

Thus the present study focused on screening PCR amplification and polymorphism of the primers using pooled chrysanthemum DNAs and then the genotyping and population structure of chrysanthemums-cultivated (Dendranthema grandiflora syn. Chrysanthemum morifolium Ramat.) and indigenous [Dendranthema zawadskii var. latilobum (Maxim.) Kitam.]-using the resulting polymorphic SSR markers.

\section{MATERIAL AND METHODS}

\section{Plant materials and DNA extraction}

In total, 95 Chrysanthemum accessions representing two species, Chrysanthemum morifolium (Nos. 1-91, 95) and D. zawadskii var. latilobum (Maxim.) Kitam. (Nos. 9294), were used to screen for SSR polymorphisms (Table 1). All accessions were kindly provided by the Chrysanthemum Research Station, Yesan, Republic of Korea. DNA was extracted from fresh leaves using the Qiagen DNA extraction kit (Qiagen, Hilden, Germany). The relative purity and concentration of extracted DNA was estimated with the NanoDrop ND-1000 (NanoDrop Technologies, Inc., Wilmington, DE, USA). The final concentration of each DNA sample was adjusted to $50 \mathrm{ng} / \mu \mathrm{L}$. 
Table 1. Information on the 95 chrysanthemums used and their inferred cluster.

\begin{tabular}{|c|c|c|c|c|}
\hline No. & Accessions & Type & Usage type & Inferred cluster \\
\hline 1 & Gukyagukhyang & Cultivated & Decoration type & 1 \\
\hline 2 & Gaeul-agassi & Cultivated & Decoration type & 1 \\
\hline 3 & Ganghohwangpaljang & Cultivated & Decoration type & 1 \\
\hline 4 & Gyeomyukbaekguk & Cultivated & Decoration type & 1 \\
\hline 5 & Gukhwagaeun & Cultivated & Decoration type & 1 \\
\hline 6 & Gukhwagiun & Cultivated & Decoration type & 1 \\
\hline 7 & Busanseol & Cultivated & Decoration type & 1 \\
\hline 8 & Okpiri & Cultivated & Decoration type & 1 \\
\hline 9 & Ibaek & Cultivated & Decoration type & 1 \\
\hline 10 & Jaunjeon & Cultivated & Decoration type & 1 \\
\hline 11 & Ipalhong & Cultivated & Decoration type & 1 \\
\hline 12 & Hongeojeon & Cultivated & Decoration type & 1 \\
\hline 13 & Cheonhyangmaehwa & Cultivated & Decoration type & 1 \\
\hline 14 & Sinma & Cultivated & Standard type & 1 \\
\hline 15 & Hi Maya & Cultivated & Standard type & 1 \\
\hline 16 & Jeong Heung Seong & Cultivated & Standard type & 1 \\
\hline 17 & Su I-Sin & Cultivated & Standard type & 1 \\
\hline 18 & Eul-nyeo & Cultivated & Standard type & 1 \\
\hline 19 & Jeong Heung Chu & Cultivated & Standard type & 1 \\
\hline 20 & Yongma & Cultivated & Standard type & 1 \\
\hline 21 & Jeong Heung Sin Nyeon & Cultivated & Standard type & 1 \\
\hline 22 & ST09-173-01 & Cultivated & Standard type & Admixture \\
\hline 23 & Baekgwang & Cultivated & Standard type & 1 \\
\hline 24 & Baekseon & Cultivated & Standard type & 1 \\
\hline 25 & Baekma & Cultivated & Standard type & 2 \\
\hline 26 & Seinonami & Cultivated & Standard type & 2 \\
\hline 27 & Yuka & Cultivated & Standard type & 2 \\
\hline 28 & Jeongju & Cultivated & Standard type & 2 \\
\hline 29 & Seikonoilse & Cultivated & Standard type & 2 \\
\hline 30 & Jangsuhwang & Cultivated & Standard type & Admixture \\
\hline 31 & Seinoisami & Cultivated & Standard type & 2 \\
\hline 32 & ST06-03-01 & Cultivated & Standard type & 2 \\
\hline 33 & ST09-148-04 & Cultivated & Standard type & 1 \\
\hline 34 & ST09-50-01 & Cultivated & Standard type & 1 \\
\hline 35 & ST09-40-02 & Cultivated & Standard type & 1 \\
\hline 36 & ST09-149-01 & Cultivated & Standard type & 1 \\
\hline 37 & ST09-136-01 & Cultivated & Standard type & 1 \\
\hline 38 & ST09-99-02 & Cultivated & Standard type & 1 \\
\hline 39 & ST09-173-11 & Cultivated & Standard type & 1 \\
\hline 40 & Gold coast & Cultivated & Spray type & 1 \\
\hline 41 & Green Day & Cultivated & Spray type & Admixture \\
\hline 42 & Monaliza & Cultivated & Spray type & 2 \\
\hline 43 & Shamlook & Cultivated & Spray type & 2 \\
\hline 44 & Aniasia & Cultivated & Spray type & 2 \\
\hline 45 & Artic Queen & Cultivated & Spray type & 2 \\
\hline 46 & Euro & Cultivated & Spray type & 2 \\
\hline 47 & Besu Bio & Cultivated & Spray type & 1 \\
\hline 48 & Sterion & Cultivated & Spray type & 2 \\
\hline 49 & Yes Together & Cultivated & Spray type & 1 \\
\hline 50 & Nice & Cultivated & Spray type & 2 \\
\hline 51 & Loyal Aridillo & Cultivated & Spray type & 2 \\
\hline 52 & Yes Nuri & Cultivated & Spray type & 2 \\
\hline 53 & Arigos & Cultivated & Spray type & 2 \\
\hline 54 & Yes Life & Cultivated & Spray type & 1 \\
\hline 55 & Penny Line & Cultivated & Spray type & 2 \\
\hline 56 & Puma & Cultivated & Spray type & 2 \\
\hline 57 & luli puf & Cultivated & Spray type & 2 \\
\hline 58 & Yes Miso & Cultivated & Spray type & 2 \\
\hline 59 & Chilo & Cultivated & Spray type & 2 \\
\hline 60 & Yes Day & Cultivated & Spray type & 2 \\
\hline 61 & Frog & Cultivated & Spray type & Admixture \\
\hline 62 & Ligan Orange & Cultivated & Spray type & Admixture \\
\hline
\end{tabular}

Continued on next page 


\begin{tabular}{|c|c|c|c|c|}
\hline No. & Accessions & Type & Usage type & Inferred cluster \\
\hline 63 & Marble Orange & Cultivated & Spray type & 2 \\
\hline 64 & Mujigae & Cultivated & Spray type & 2 \\
\hline 65 & Moon Light & Cultivated & Spray type & 2 \\
\hline 66 & Biking & Cultivated & Spray type & 2 \\
\hline 67 & Bophi & Cultivated & Spray type & 2 \\
\hline 68 & Borami & Cultivated & Spray type & 2 \\
\hline 69 & Bimini & Cultivated & Spray type & Admixture \\
\hline 70 & Yes Swan & Cultivated & Spray type & Admixture \\
\hline 71 & Yes Line & Cultivated & Spray type & 2 \\
\hline 72 & Yes Morning & Cultivated & Spray type & 2 \\
\hline 73 & Uno Ivory & Cultivated & Spray type & 2 \\
\hline 74 & Yes Star & Cultivated & Spray type & 2 \\
\hline 75 & Hwiparam & Cultivated & Spray type & 2 \\
\hline 76 & Green Berry & Cultivated & Pot mum & Admixture \\
\hline 77 & Leema honey & Cultivated & Pot mum & Admixture \\
\hline 78 & Monroe & Cultivated & Pot mum & Admixture \\
\hline 79 & Peace Angel & Cultivated & Pot mum & 3 \\
\hline 80 & Peace Yellow & Cultivated & Pot mum & 3 \\
\hline 81 & Peace Pink & Cultivated & Pot mum & 3 \\
\hline 82 & Saskia & Cultivated & Pot mum & 3 \\
\hline 83 & Phikho Salto & Cultivated & Pot mum & 3 \\
\hline 84 & Peace Copper & Cultivated & Pot mum & 3 \\
\hline 85 & Phasoa Red & Cultivated & Pot mum & 3 \\
\hline 86 & Geumbangul & Cultivated & Garden mum & 3 \\
\hline 87 & Dohwa Ball & Cultivated & Garden mum & 3 \\
\hline 88 & Popcorn Ball & Cultivated & Garden mum & 3 \\
\hline 89 & Bright Ball & Cultivated & Garden mum & 2 \\
\hline 90 & $\mathrm{G}-20$ & Cultivated & Garden mum & 3 \\
\hline 91 & $\mathrm{G}-28$ & Cultivated & Garden mum & 3 \\
\hline 92 & Gujeolcho & Indigenous & & 3 \\
\hline 93 & Ulreung Gukhwa & Indigenous & & 3 \\
\hline 94 & Gwandong Gukhwa & Indigenous & & 3 \\
\hline 95 & G31 & Cultivated & Garden mum & 3 \\
\hline
\end{tabular}

\section{SSR marker screening}

In total, 100 SSR primer pairs designed by Moe et al. (2011) were screened for polymorphisms using the SSR MANAGER program (Kim, 2004); 40 DNA samples were used to screen the PCR amplification of the 100 designed primer pairs. PCR products were examined by electrophoresis (3\% agarose gel). Of the 100 primer pairs, 12 were successful in providing PCR products. The M13-tail PCR method of Schuelke (2000) was used to measure the size of the PCR products. Forward sequences of the 12 primers were attached to the M13-tail (TGTAAAACGACGGCCAGT), and PCRs were run together with the respective normal reverse primers labeled with fluorescent dyes (FAM, VIC, NED and PET). The fragments of PCR products were analyzed with the ABI-3500 Genetic Analyzer (Applied Biosystems, Foster City, CA, USA) using the GeneMapper 4.1 software (Applied Biosystems) and sized precisely using the GeneScan 600 LIZ Size Standard v2.0 (Applied Biosystems). Conditions for PCR amplification were $94^{\circ} \mathrm{C}$ for 3 min, 35 cycles each at $94^{\circ} \mathrm{C}$ for $30 \mathrm{~s}, 55^{\circ} \mathrm{C}$ (varied according to annealing temperature requirements of primers in Table 2) for $45 \mathrm{~s}$, and $72^{\circ} \mathrm{C}$ for $1 \mathrm{~min}$, followed by a final extension at $72^{\circ} \mathrm{C}$ for $10 \mathrm{~min}$. 
Table 2. Characterization of 12 novel simple sequence repeat markers.

\begin{tabular}{|c|c|c|c|c|}
\hline No. & ID & Forward (above) and reverse (below) & Repeat motif & $\mathrm{A}_{\mathrm{T}}{ }^{1}\left({ }^{\circ} \mathrm{C}\right)$ \\
\hline 1 & KNUCRY-10 & $\begin{array}{l}\text { GTGTCTTCATCCCACCACCA } \\
\text { TGTGAGAGAGTGAGTGTAGTGTGAG }\end{array}$ & $(\mathrm{CA})_{24}$ & 60 \\
\hline 2 & KNUCRY-16 & $\begin{array}{l}\text { TGTTCACCCATTCACAGCTC } \\
\text { CACATGTATGACTAGGTGAGGTGA }\end{array}$ & $(\mathrm{AC})_{3},(\mathrm{AC})_{4}$ & 53 \\
\hline 3 & KNUCRY-35 & $\begin{array}{l}\text { CCTCGCACTACTTCCAAATGA } \\
\text { GGAGATTGTTTGTTCGTATCCTT }\end{array}$ & $(\mathrm{CA})_{27},(\mathrm{CA})_{16}$ & 50 \\
\hline 4 & KNUCRY-58 & $\begin{array}{l}\text { GGTGGAATTGCTCCTTGTTG } \\
\text { CCATCATCAACACAAGCTTCA }\end{array}$ & $(\mathrm{TTG})_{5},(\mathrm{TTG})_{6}$ & 60 \\
\hline 5 & KNUCRY-59 & $\begin{array}{l}\text { CGGTCCTCTCAGCCTTATTG } \\
\text { GGTGTGTGTGTGAAGGTGCT }\end{array}$ & $(\mathrm{CA})_{5}$ & 57 \\
\hline 6 & KNUCRY-75 & $\begin{array}{l}\text { GTGGTTGAGCCATTTGAGGT } \\
\text { TTGGCTATTGTGATTTCTACGC }\end{array}$ & $(\mathrm{TTG})_{7}$ & 55 \\
\hline 7 & KNUCRY-76 & $\begin{array}{l}\text { TTGAGGTTGTGGAAATGCAG } \\
\text { CGCGTTAACTTTGGTGTTTTT }\end{array}$ & $(\mathrm{TTG})_{7}$ & 55 \\
\hline 8 & KNUCRY-77 & $\begin{array}{l}\text { CCCGGTTATCATGTGTATGC } \\
\text { CGTATTTAAAGGTTTTCCTTTCG }\end{array}$ & $(\mathrm{CACG})_{3},(\mathrm{CA})_{28}$ & 50 \\
\hline 9 & KNUCRY-84 & $\begin{array}{l}\text { CTAGGCTCCTTCAGCCCTCT } \\
\text { TCTGGACTAGCCGTCAGTTG }\end{array}$ & $(\mathrm{CA})_{15}$ & 50 \\
\hline 10 & KNUCRY-85 & $\begin{array}{l}\text { GACCAACAAAACGGAATGCT } \\
\text { GTTGTCGTCCGTTGGCTAGT }\end{array}$ & $(\mathrm{CA})_{4}$ & 50 \\
\hline 11 & KNUCRY-94 & $\begin{array}{l}\text { CATTCAGGACAGTCATACAAGTG } \\
\text { CAACACACACACACACAGGAAT }\end{array}$ & $(\mathrm{GAA})_{4}$ & 55 \\
\hline 12 & KNUCRY-98 & $\begin{array}{l}\text { TCACATCACACATCACTGCAA } \\
\text { TGTGTGTGAGGGACACATGA }\end{array}$ & $(\mathrm{AC})_{4}$ & 55 \\
\hline
\end{tabular}

${ }^{1}$ Annealing temperature.

\section{Genotyping of 95 Chrysanthemum accessions}

Twelve newly identified polymorphic SSR markers were used for genotyping and population structure analysis of 95 Chrysanthemum accessions. The SSR alleles of 12 markers were resolved on a 3500 Genetic Analyzer (Applied Biosystems). The variability at each locus was measured in terms of the number of alleles, rare alleles and specific alleles, genotype number, heterozygosity $(\mathrm{H})$, major allele frequency (MAF), gene diversity (GD), and polymorphic information content (PIC). These variables were measured in terms of the shared allele frequencies using PowerMarker 3.25 (Liu and Muse, 2005). The neighbor- joining method was used to construct a phylogram from a distance matrix using MEGA4 software (Tamura et al., 2007) embedded in PowerMarker. POPGENE (ver. 1.32) (Yeh et al., 1999) was used to test for Hardy-Weinberg equilibrium (HWE).

\section{Population structure analysis}

The model-based program Structure 2.2 (Pritchard et al., 2000; Falush et al., 2003) was used to determine the population structure for 95 accessions using a burn-in of 100,000, a run length of 200,000, and a model allowing for admixture and correlated allele frequencies. At least three runs of the Structure program were performed by setting the number of populations $(K)$ from 2 to 10 . The model choice criterion to detect the most probable value of $K$ was $\Delta \mathrm{K}$, an ad hoc quantity related to the second-order change of the log probability of data with respect to the number of clusters inferred in Structure (Evanno et al., 2005). 


\section{RESULTS}

\section{SSR polymorphisms}

In total, 200 alleles were detected, with an average of 16.7 alleles per locus. KNUCRY-84 produced the highest number of alleles (35), whereas only four alleles were observed at KNUCRY-10 and KNUCRY-75 (Table 3). Allele size ranged from 134 to $500 \mathrm{bp}$. The allele frequency database showed that rare alleles (with a frequency $<0.05$ ) comprised $77.5 \%$ of all alleles, while intermediate $(0.05<$ frequency $<0.50)$ and abundant alleles (frequency $>0.50$ ) comprised 19.5 and $3 \%$, respectively, of all detected alleles, showing that most alleles were of low frequency (Figure 1). The specific and rare alleles detected ranged from 0 to 18 and 1 to 30 , respectively, and the maximum numbers of both alleles were detected with KNUCRY-84. Values for heterozygosity ranged from 0.187 to 0.926 (mean, 0.588 ). The average gene diversity and PIC values were 0.662 and 0.635 , with a range from 0.229 (KNUCRY-10) to 0.888 (KNUCRY-77) and from 0.218 (KNUCRY-10) to 0.879 (KNUCRY-77), respectively. Eleven loci deviated from HWE and showed highly significant linkage disequilibrium $(\mathrm{P}<0.01$; Table 3). A correlation analysis revealed that allelic richness was significantly and positively associated with gene diversity $(r=0.743, \mathrm{P}<0.01)$ and $\mathrm{PIC}$ value $(r=0.776, \mathrm{P}<0.01$; data not shown). Moe et al. (2010), however, found no significant correlation between allelic richness and the number of repeats in the SSR loci.

\begin{tabular}{|c|c|c|c|c|c|c|c|c|c|}
\hline Marker & Size range (bp) & $N_{\mathrm{A}}{ }^{1}$ & $\mathrm{MAF}^{2}$ & $\mathrm{RA}^{3}$ & $\mathrm{SA}^{4}$ & $\mathrm{GD}^{5}$ & $\mathrm{H}^{6}$ & $\mathrm{PIC}^{7}$ & $\mathrm{P}^{8}$ \\
\hline KNUCRY-10** & $160-410$ & 4 & 0.874 & 2 & 1 & 0.229 & 0.187 & 0.218 & 0.0057 \\
\hline KNUCRY-16** & $151-500$ & 29 & 0.382 & 27 & 18 & 0.742 & 0.236 & 0.708 & 0.0000 \\
\hline KNUCRY-35** & $160-435$ & 14 & 0.315 & 8 & 5 & 0.818 & 0.865 & 0.797 & 0.0000 \\
\hline KNUCRY-58** & $150-254$ & 5 & 0.620 & 1 & 1 & 0.548 & 0.759 & 0.495 & 0.0007 \\
\hline KNUCRY-59** & $134-500$ & 27 & 0.437 & 23 & 11 & 0.779 & 0.517 & 0.767 & 0.0000 \\
\hline KNUCRY-75** & $165-293$ & 4 & 0.516 & 1 & 0 & 0.621 & 0.926 & 0.555 & 0.0000 \\
\hline KNUCRY-76 & $150-286$ & 8 & 0.817 & 6 & 5 & 0.315 & 0.367 & 0.290 & 0.9999 \\
\hline KNUCRY-77** & $257-390$ & 31 & 0.213 & 27 & 12 & 0.888 & 0.632 & 0.879 & 0.0000 \\
\hline KNUCRY-84** & $152-500$ & 35 & 0.332 & 30 & 18 & 0.843 & 0.348 & 0.832 & 0.0000 \\
\hline KNUCRY-85** & $157-268$ & 13 & 0.403 & 7 & 3 & 0.773 & 0.682 & 0.749 & 0.0000 \\
\hline KNUCRY-94** & $160-197$ & 7 & 0.617 & 4 & 1 & 0.566 & 0.713 & 0.524 & 0.0000 \\
\hline KNUCRY-98** & $157-457$ & 23 & 0.335 & 19 & 9 & 0.824 & 0.819 & 0.807 & 0.0000 \\
\hline Mean & & 16.7 & 0.488 & 5 & 7 & 0.662 & 0.588 & 0.635 & \\
\hline
\end{tabular}

${ }^{1}$ Number of alleles; ${ }^{2}$ major allele frequency; ${ }^{3}$ rare allele; ${ }^{4}$ specific allele; ${ }^{5}$ gene diversity; ${ }^{6}$ heterozygosity; ${ }^{7}$ polymorphic information content; ${ }^{8}$ probability value of chi-square test for HWE. **Loci deviating from HardyWeinberg equilibrium (HWE) at the 0.01 significance level.

\section{Genetic relationship and cluster analysis}

A genetic distance-based analysis was performed to evaluate the usefulness of these newly developed SSRs for the study of genetic variations and phylogenetic relationships among a large germplasm collection of chrysanthemums. To determine the population structure of 95 accessions of Chrysanthemum, the model-based program Structure was used. Although the distribution of L(K) showed the highest peak, $K=6$ (Figure 2a), clusters could not be differentiated because one cluster showed inferred values that were lower than $70 \%$. Also, the slope at $K=3$ was sharper and steeper than that at $K=6$. Thus, when a further ad hoc quantity $(\Delta K)$ was used to overcome the difficulty of 
interpreting the real $K$ values (Evanno et al., 2005), the highest value of $\Delta K$ for the 95 accessions was found to be at $K=3$ (Figure 2b). The Chrysanthemum accessions tested were distributed into three clusters with $89.4 \%$ (shared $>70 \%$ ) membership ( 85 accessions: 34 for cluster 1, 35 for cluster 2 , 16 for cluster 3) showing 10.6\% admixture (10 accessions) (Figure 3, Table 1). All decoration-type accessions and most standard-type accessions were found together in cluster 1. Among the standardtype accessions, some were found to be members of cluster 2. Most of the spray-type accessions belonged to cluster 2 and some were related to cluster 1. Three indigenous accessions (Gujeolcho, Ulreung Gukhwa and Gwandong Gukhwa), pot mums and garden mums, were found in cluster 3.

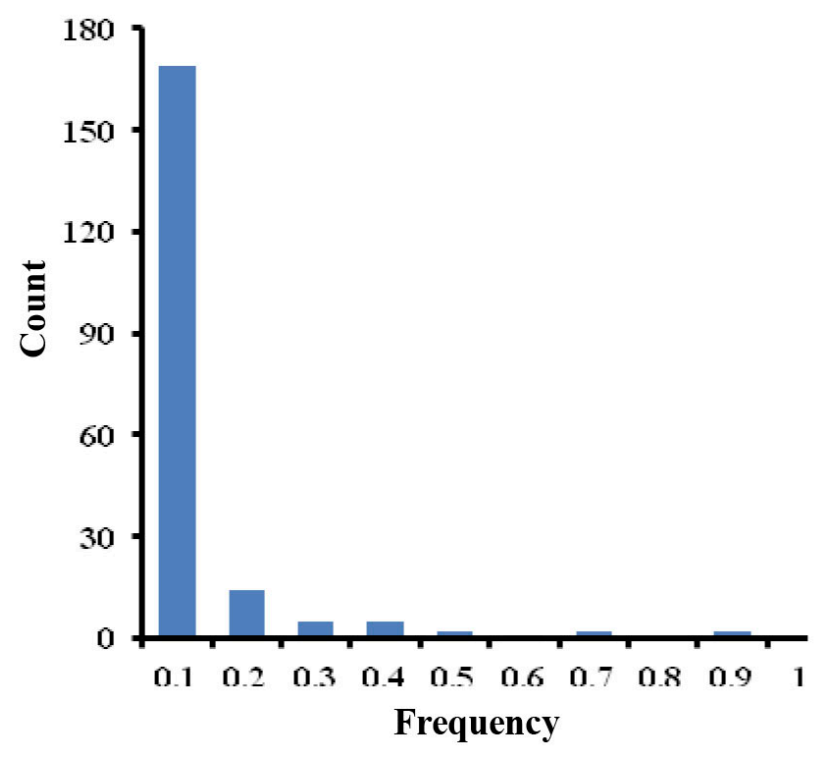

Figure 1. Histogram of allele frequencies for 200 alleles in the 95 chrysanthemum accessions.

A

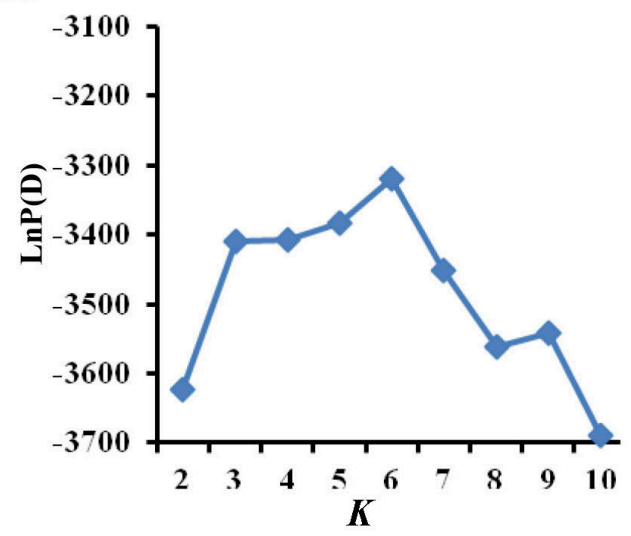

B

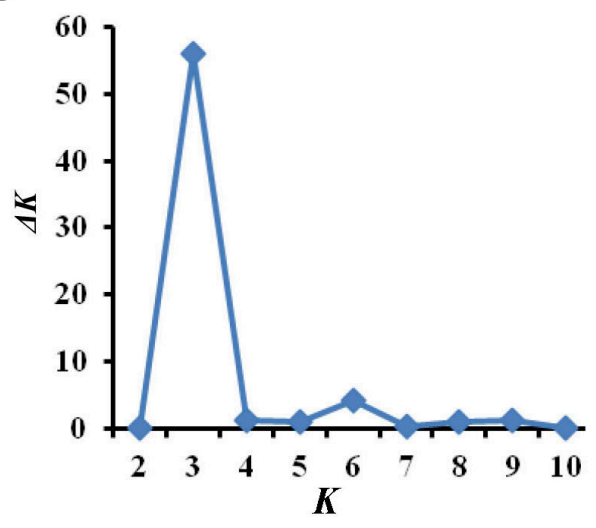

Figure 2. A. Likelihood of the data $(\mathrm{N}=95), \mathrm{L}(\mathrm{K})$, as a function of $K$ (the number of groups used to stratify the sample) and $\mathbf{B}$. values of $\Delta K$, with its modal value detecting a true $K$ of three groups $(K=3)$. 


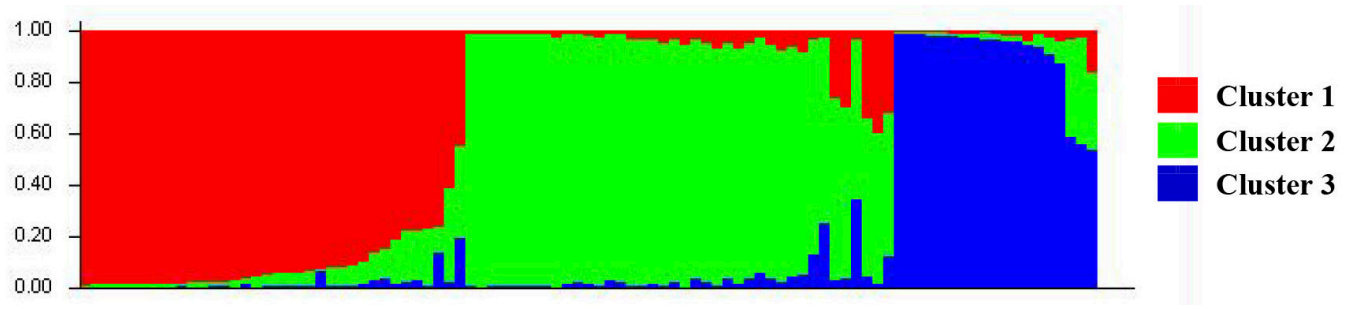

Figure 3. Model-based clustering for each of the 95 chrysanthemum accessions examined based on the 12 SSR markers used to build the Q matrix. Each accession is divided into several hypothetical populations based on the proportional membership.

The genetic distance between cluster 1 and cluster $3(0.074)$ was the highest among the relationships of the populations, followed by the distance between cluster 2 and cluster 3 (0.058), and the lowest genetic distance (0.026) was between cluster 1 and cluster 2 (Table $4)$. The average genetic distance within the same population was highest in cluster $1(0.638)$ and lowest in cluster $3(0.596)$. The genetic diversity in each model-based population was measured (Table 5). Reduced genetic diversity, as measured by differences in the number of alleles, genetic diversity, and PIC values, was detected within subpopulations in comparison with the overall population. Among three subpopulations, cluster 1 showed the highest genetic parameter values except in heterozygosity, and cluster 3 showed the lowest values in all parameters.

Table 4. Allele frequency divergence among clusters (net nucleotide distance, below diagonal) and average distance (expected heterozygosity) between individuals in same cluster (diagonal).

\begin{tabular}{lccc}
\hline & Cluster 1 & Cluster 2 & Cluster 3 \\
\hline Cluster 1 & 0.683 & & \\
Cluster 2 & 0.026 & 0.663 & 0.596 \\
Cluster 3 & 0.074 & 0.058 & \\
\hline
\end{tabular}

Table 5. Genetic diversity of model-based populations for 95 chrysanthemum accessions.

\begin{tabular}{lccccc}
\hline & Sample size & $N_{\mathrm{A}}{ }^{1}$ & $\mathrm{GD}^{2}$ & $\mathrm{H}^{3}$ & $\mathrm{PIC}^{4}$ \\
\hline Overall & 95 & 16.7 & 0.662 & 0.588 & 0.635 \\
Cluster 1 & 34 & 10.2 & 0.639 & 0.596 & 0.608 \\
Cluster 2 & 35 & 9.3 & 0.614 & 0.652 & 0.580 \\
Cluster 3 & 16 & 4.6 & 0.549 & 0.397 & 0.500 \\
\hline
\end{tabular}

${ }^{1}$ Number of alleles; ${ }^{2}$ gene diversity; ${ }^{3}$ heterozygosity; ${ }^{4}$ polymorphic information content.

A GD-based analysis was performed by calculating the shared allele frequencies among the 95 accessions, and an unrooted phylogram (neighbor-joining tree) was computed using Powermarker 3.23 and MEGA4 (Tamura et al., 2007). A clustering pattern similar to that of the Structure analysis was observed (Figure 4). Colors were applied according to the model-based cluster analysis results. 


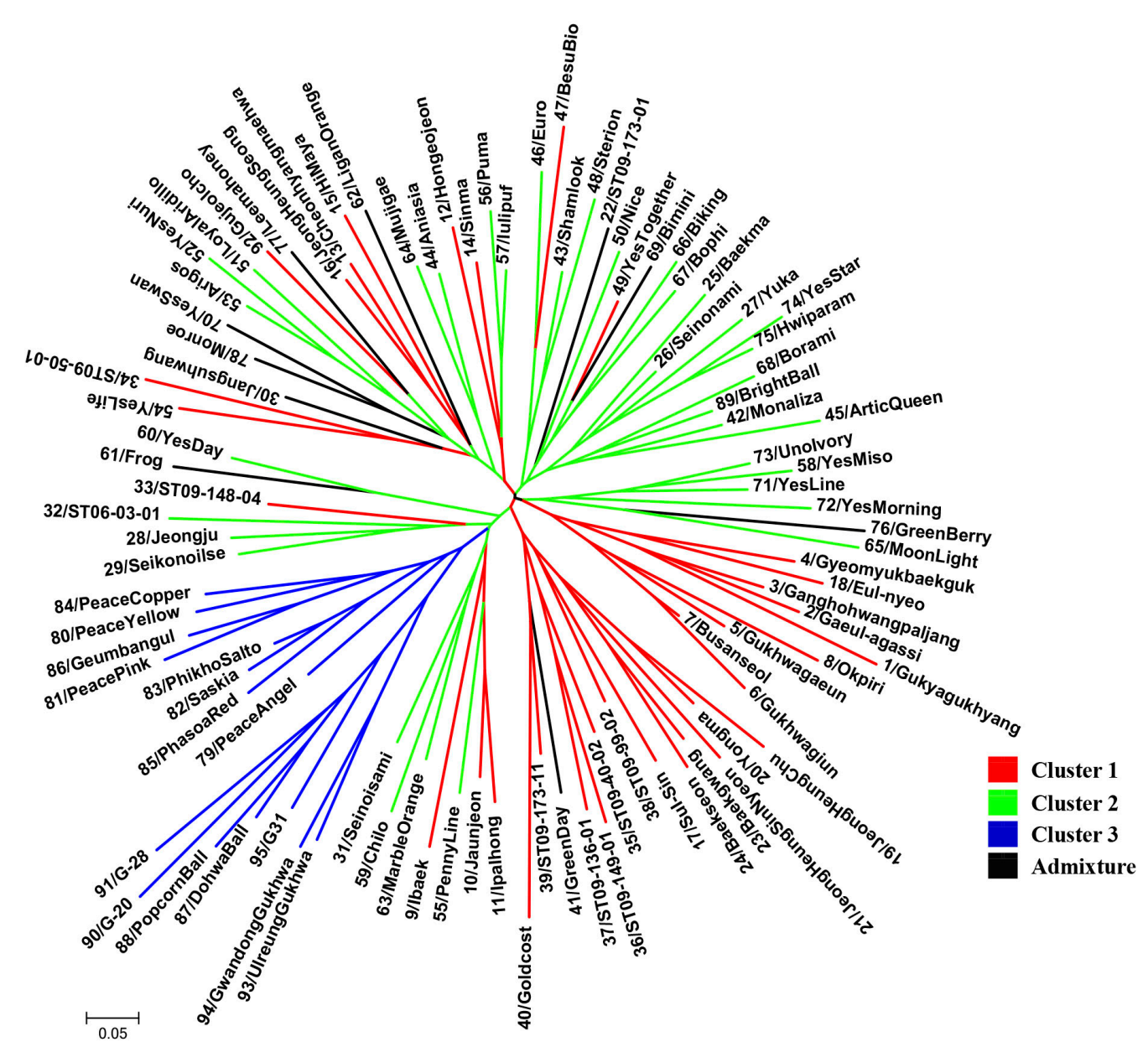

Figure 4. Neighbor-joining tree of 95 accessions using shared allele distances among 12 SSR markers.

\section{DISCUSSION}

Chrysanthemum is one of the most important flowers worldwide, not only for ornamental purposes, but also for its medicinal utility. Because it has the ability to survive in and is adaptable to different ecosystems, chrysanthemums spread easily. It was domesticated long ago and artificial crossings have led to numerous cultivars. The genome of the chrysanthemum is composed of multiple sets of chromosomes that range from diploid to decaploid (Hartl and Jones, 2009). Moreover, the chrysanthemum has a strong self-incompatibility system, which leads to gene flow and makes for a genome complex. Difficulties in systematically classifying chrysanthemum were reported by Yang et al. (2006), who stated that lineage recombination was due to extensive sharing of chloroplast haplotypes among tetraploid populations of different origins. The characterization of chrysanthemums is vital for their conservation and management as well as for the understanding of their genetic relationships. 
Several DNA works and genetic studies have investigated the chrysanthemum using different molecular markers, such as RAPDs (Wolff et al., 1995; Chatterjee et al., 2006; Zhang et al., 2010b), sequence-characterized amplified regions (Chatterjee et al., 2006), chloroplast SSR markers (Yang et al., 2006), amplified fragment length polymorphisms (Zhang et al., 2010b), and sequence-related amplified polymorphisms (Zhang et al., 2011). However, the results based on morphology, cytology, interspecific hybridization, and molecular systematics have indicated that a lot of work remains to be done on chrysanthemums (Silan et al., 2002). Thus, an intensive classification of inter- and intraspecies variation should be performed.

Moe et al. (2011) identified the distribution of SSR motifs in a chrysanthemum SSRenriched library through 454 pyrosequencing technology to develop a more powerful analytical tool, SSR markers. Based on their work, 100 SSRs were developed in the present study and screened for polymorphisms. Twelve of the 100 SSR markers designed were polymorphic. KNUCRY-77 showed the greatest polymorphism and KNUCRY-10 showed the lowest polymorphisms among the 12 SSR loci. The wide variation in gene diversity, heterozygosity, rare alleles, and specific alleles revealed by SSRs among the different Chrysanthemum accessions reflected high polymorphism in the accessions of chrysanthemums. In the present study, three indigenous accessions were also used to detect the cross-amplification ability of the loci. These 12 noble SSR loci were found to be cross-amplified. However, intensive genotyping with many indigenous accessions is still needed to confirm the wide potential applicability of these loci for the study of interspecific genetic diversity.

In a model-based population, the Chrysanthemum accessions tested were distributed into three clusters, with $89.4 \%$ (shared $>70 \%$ ) membership showing $10.6 \%$ admixture. The high value of admixture indicates ancestral relatedness among the accessions tested. Almost all accessions having the same plant type clustered together, except spray types. Decoration-type accessions and most of the standard accessions were found in cluster 1. In Korea, the decoration type is popular for flower arrangements or training the plant to have a desired shape, such as a butterfly, star, or country map. Decoration types used in the present study were similar to the standard type in terms of flower size and plant type. Most of the spray types grouped in cluster 2 and some were related together in cluster 1. Flower sizes of spray types range from small to medium and some standard types have medium-sized flowers. This may be the reason why the standard type and spray type were found in cluster 1 and cluster 2. Pot mum and garden mum types were found together with three indigenous accessions in cluster 3. Generally, the pot mum type is a short plant type having many branches and many small- to medium-sized flowers, and the garden mum is a bush type with small-sized flowers on the whole plant. Based on the phenotype and cluster relationship, these 12 SSRs may be related to flower size and plant-architecture phenotypes.

According to the results of genetic distance, cluster 1 and cluster 3 had the highest genetic distance between them, and the phenotypes of the accessions between these two clusters were significantly different. Genetic diversity values for each subpopulation showed that cluster 1 and cluster 2 had greater genetic diversity values, suggesting more allelic richness and more diverse genotypes in these two clusters.

The distance-based dendrogram was basically similar to the clustering by modelbased structure analysis. The SSRs of the present study were successfully used to measure genetic diversity and relationships within the chrysanthemum collection, and could be useful for determining genetic diversity, identification, conservation, core set construction, and crop improvement of chrysanthemum germplasm. 


\section{ACKNOWLEDGMENTS}

Research supported by the Cooperative Research Program for Agriculture Science \& Technology Development (Project \#PJ007884), Rural Development Administration, Republic of Korea.

\section{REFERENCES}

Anderson NO, Ascher PD and Widmer RE (1992). Inbreeding depression in garden and glasshouse chrysanthemums: germination and survivorship. Euphytica 62: 155-169.

Chatterjee J, Mandal AKA, Ranade SA, Silva JATD, et al. (2006). Molecular systematics in Chrysanthemum grandiflorum (Ramat.) Kitamura. Sci. Hortic. 110: 373-378.

Chen FD, Jiang JF and Guo WM (2003). Heredity of several flower characters in Dendranthema grandiflorum with small inflorescences. Acta Hortic. Sin. 30: 175-182.

Cho YI, Chung JW, Lee GA, Ma KH, et al. (2010). Development and characterization of twenty-five new polymorphic microsatellite markers in proso millet (Panicum miliaceum L.). Genes Genomics 32: 267-273.

Cho YI, Park JH, Lee CW, Ra WH, et al. (2011). Evaluation of the genetic diversity and population structure of sesame (Sesamum indicum L.) using microsatellite markers. Genes Genomics 33: 187-195.

Dixit A, Chung JW, Zhao WG, Lee GA, et al. (2010). Development of new microsatellite markers for molecular diversity analysis of Citrus species. J. Hortic. Sci. Biotechnol. 85: 521-527.

Evanno G, Regnaut S and Goudet J (2005). Detecting the number of clusters of individuals using the software STRUCTURE: a simulation study. Mol. Ecol. 14: 2611-2620.

Falush D, Stephens M and Pritchard JK (2003). Inference of population structure using multilocus genotype data: linked loci and correlated allele frequencies. Genetics 164: 1567-1587.

Gupta P and Varshney R (2000). The development and use of microsatellite markers for genetic analysis and plant breeding with emphasis on bread wheat. Euphytica 113: 163-185.

Hartl DL and Jones EW (2009). Genetics: Analysis of Genes and Genomes. Jones and Bartlett Publishers, Burlington.

Huang SC, Tsai CC and Sheu CS (2000). Genetic analysis of Chrysanthemum hybrids based on RAPD molecular markers. Bot. Bull. Acad. Sin. 41: 257-262.

Jiang JF, Chen FD and Guo WM (2003). Heredity of several characters in Dendranthema grandiflorum with small inflorescences. J. Nanjing Agric. Univ. 26: 11-15.

Khaing AA, Moe KT, Chung JW, Baek HJ, et al. (2013). Genetic diversity and population structure of the selected core set in Amaranthus using SSR markers. Plant Breed. 132: 165-173.

Kim KW (2004). Developing One-Step Program (SSR MANAGER) for Rapid Identification of Clones with SSRs and Primer Designing. Master's thesis, Seoul National University, Seoul.

Li MT, Yu LJ, Wang LM, Liu JM, et al. (2005).The heredity of flower colors and the discovery of flower color chimera in chrysanthemum species. Yi. Chuan 27: 948-952.

Liu K and Muse SV (2005). PowerMarker: an integrated analysis environment for genetic marker analysis. Bioinformatics 21: 2128-2129.

Lu FH and Park YJ (2012). An SNP downstream of the OsBEIIb gene is significantly associated with amylase content and viscosity properties in rice (Oryza sativa L.). J. Cereal Sci. 56: 706-712.

Moe KT, Zhao W, Song HS, Kim YH, et al. (2010). Development of SSR markers to study diversity in the genus Cymbidium. Biochem. Syst. Ecol. 38: 585-594.

Moe KT, Ra SB, Lee GA, Lee MC, et al. (2011). Distribution and frequency of SSR motifs in the Chrysanthemum SSRenriched library through 454 pyrosequencing technology. Korean J. Int. Agric. 23: 546-551.

Moe KT, Kwon SW and Park YJ (2012). Trends in genomics and molecular marker systems for the development of some underutilized crops. Genes Genomics 34: 451-466.

Pritchard JK, Stephens M and Donnelly P (2000). Inference of population structure using multilocus genotype data. Genetics 155: 945-959.

Richards AJ (1986). Plant Breeding Systems. George Allen and Unwin, London.

Schuelke M (2000). An economic method for the fluorescent labeling of PCR fragments. Nat. Biotechnol. 18: 233-234.

Silan D, Wenkui W and Jiaping H (2002). Advances of researches on phylogeny of Dendranthema and origin of Chrysanthemum. J. Beijing For. Univ. 24: 230-234.

Tamura K, Dudley J, Nei M and Kumar S (2007). MEGA4: Molecular evolutionary genetics analysis (MEGA) software 
version 4.0. Mol. Biol. Evol. 24: 1596-1599.

Wolff K and Peters-van Rijn J (1993). Rapid detection of genetic variability in chrysanthemum (Dendranthema grandiflora Tzvelev) using random primers. Heredity 71: 335-341.

Wolff K, Zietiewicz E and Hofstra H (1995). Identification of chrysanthemum cultivars and stability of DNA fingerprint patterns. Theor. Appl. Genet. 91: 439-447.

Yang W, Glover BJ, Rao GY and Yang J (2006). Molecular evidence for multiple polyploidization and lineage recombination in the Chrysanthemum indicum polyploid complex (Asteraceae). New Phytol. 171: 875-886.

Yeh F, Yang R and Boyle T (1999). POPGENE Version 1.32 Microsoft Windows-Based Freeware for Population Genetic Analysis. University of Alberta, Edmonton, Alberta.

Yeung HC (1983). Handbook of Chinese Herbs. Institute of Chinese Medicine, Los Angeles.

Yoon MY, Moe KT, Kim DY, Rho RI, et al. (2012). Genetic diversity and population structure analysis of strawberry (Fragaria x ananassa Duch.) using SSR markers. Electron. J. Biotechnol. 15: 71-85.

Zhang F, Fang WM, Chen FD and Chen SM (2010a). Combining ability analysis on ornamental characters of Chrysanthemum. Acta Hort. Sin. 37: 589-596.

Zhang F, Chen S, Chen F, Fang W, et al. (2010b). A preliminary genetic linkage map of chrysanthemum (Chrysanthemum morifolium) cultivars using RAPD, ISSR and AFLP markers. Sci. Hortic. 125: 422-428.

Zhang F, Chen S, Chen F, Fang W, et al. (2011). Genetic analysis and associated SRAP markers for flowering traits of chrysanthemum (Chrysanthemum morifolium). Euphytica 117: 15-24.

Zhao JY, Chen FD, Teng NJ and Chen SM (2009). Genetic analysis and RAPD marker of creeping habits in groundcover chrysanthemum. Sci. Agric. Sin. 42: 734-741.

Zhao WG, Lee GA, Kwon SW, Ma KH, et al. (2012). Development and use of novel SSR markers for molecular genetic diversity in Italian millet (Setaria italica L.). Genes Genomics 34: 51-57. 